\title{
INEQUIDADES INVISIBLES EN LA EDUCACIÓN CHILENA: BRECHAS ENTRE ESTUDIANTES URBANOS Y RURALES EN LA PRUEBA PISA DE LECTURA (2009)*
}

\author{
SEBASTIÁN Donoso-Díaz \\ ÓSCAR ARIAS-ROJAS ${ }^{* * *}$ \\ Carla Gajardo Poblete*** \\ Claudio Frites CAMILLA ${ }^{* * * *}$
}

\begin{abstract}
RESUMEN: El estudio da cuenta de diferenciasrelevantes en materia de resultados de las Pruebas Pisa de Lenguaje 2009 de estudiantes que viven en sectores rurales en comparación con los que no. Establecido a partir de un análisis jerárquico multinivel de las variables de sus bases de datos, identificando dos niveles: (i) hogar/estudiante y (ii) establecimiento. Los principales resultados confirman que el medio rural ofrece menos oportunidades que el medio no rural, incidiendo en que los estudiantes rurales se vean desfavorecidos en sus logros académicos, entre otros motivos por acumulación histórica (y sinérgica) de inequidades. Los resultados muestran que se debiesen implementar políticas compensatorias en algunas variables determinantes, a saber: acceso y permanencia en la educación preescolar, reducción de la repitencia, estrategias de escolarización de las madres, etc. que tendrían un impacto positivo sobre los resultados de los estudiantes rurales, reduciendo en forma importante las grandes inequidades actuales.
\end{abstract}

Palabras claves: Prueba Pisa. Brechas rural-urbano. Inequidad.

\footnotetext{
* Una versión preliminar de este trabajo se presentó en el Primer Congreso Latinoamericano de Medición y Evaluación Educacional (Colmee), Santiago de Chile, 23 y 24 de octubre de 2012. Equipo de investigación del Instituto de Investigación y Desarrollo Educacional de la Universidad de Talca (Chile). Este trabajo es parte de los Proyectos DI 006050 financiado por la Dirección de Investigación de la Universidad de Talca, y Conicyt MEC - 80010006, se agradece a Claude Auroi sus aportes y comentarios.

** Universidad de Talca. Talca - Chile.

*** Universidad de Talca. Talca - Chile.

*** Universidad de Talca. Talca - Chile.

***** Universidad de Talca. Talca - Chile.

Contato com os autores: <donoso.seb@gmail.com>
} 
Inequidades invisibles en la educación Chilena...

\title{
INVisible INEQUALITIES IN CHILEAN EDUCATION: GAPS BETWEEN URBAN AND RURAL STUdENTS IN PISA'S REAding LITERACY ASSESSMENT (2009)
}

\begin{abstract}
This article compares the Pisa 2009 Reading Literacy test results of urban and rural students, through a multi-level hierarchical analysis of the database variables, identifying two levels: (i) home/student and (ii) school. The main results confirm that students in rural areas have fewer opportunities than those in urban areas, which means that rural students face disadvantages in terms of academic achievement, due to historical inequalities. The scores show that remedial policies are necessary in specific areas, such as: access and retention in preschool education, reduction of student failure rates, maternal education programs, etc., that could have a positive impact on students in rural areas, significantly reducing the current situation of inequality.
\end{abstract}

Key words: Pisa Test. Urban-rural gaps. Inequality.

\section{INIQUités INVISIBles dANS L'ÉDUCATION ChILIENNE: LACUNES ENTRE DES ÉTUDIANTS URBAINS ET RURAUX DANS L'Évaluation Pisa de lecture (2009)}

RÉSUMÉ: L'étude aborde des différences importantes en matière de résultats des Évaluations Pisa de Lecture, de 2009, d'étudiants qui vivent dans des secteurs agricoles en comparaison avec ceux qui n'y habitent pas. On prend comme point de départ une analyse hiérarchique des variables de bases de données, en identifiant deux niveaux: (i) logement/étudiant et (ii) établissement. Les principaux résultats confirment que le milieu agricole offre moins d'opportunités par rapport au milieu non agricole, par conséquent les étudiants du milieu rural se voient défavorisés dans leur performance académique, entre autres raisons par accumulation historique (et synergétique) d'iniquités. Les résultats montrent que devraient être mises en oeuvre des politiques compensatoires dans quelques variables déterminantes, à savoir: accès et permanence dans l'éducation préscolaire, réduction de rédoublement, stratégies de scolarisation des mères, entre autres, ce qui arriverait à un impact positif sur les résultats des étudiants du milieu rural, en réduisant de forme significative la grande iniquité actuelle.

Mots-clés: Évaluation Pisa. Lacunes rural-urbain. Iniquités.

\section{Introducción}

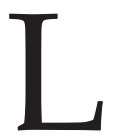

a educación es uno de los factores claves en la búsqueda de mayor equidad y justicia en la sociedad, no obstante en ocasiones las expectativas generadas en torno de ella no se corresponden con las posibilidades reales de generar los cambios deseados (TEDESCO, 2012). Los fuertes movimientos sociales que se han venido sucediendo en distintas latitudes, y desde el año 2006 en adelante con mucha fuerza en Chile, dan cuenta deuna fuerte presión y urgencia en pro de reformas por una mayor igualdad en el ámbito educativo. 
En este marco, el sistema educacional chileno, desde que se tenga memoria y datos (SALAS, 1917; LABARCA, 1939; AHUMADA, 1958; HAMUY, 1961; ILLANES, 1991; CAVIERES, 2011; SERRANO; PONCE DE LEÓN; RENGIFO, 2012a, 2012b), ha presentado una alta inequidad en los resultados entre la población que vive en las urbes respecto de la que habita en medios rurales, lo cual es reflejo de la histórica desigualdad de oportunidades que se ha observado en el mundo rural, situación que se replica también, aunque en menor medida, en relación a las oportunidades provistas en los principales centros urbanos del país con las ciudades de segundo y de tercer nivel. En razón de ello puede resultar extraño atender estas desigualdades ya que han sido "parte del paisaje pedagógico de los sistemas educativos y sociales de los países del sur del mundo", siendo consideradas normales y aceptables. Sin embargo, la percepción sobre la legitimidad de esta situación ha ido cambiando en los últimos años. Por ello, actualmente es importante poder explicar algunas desigualdades de resultados de aprendizaje que se producen entre los escolares del sector rural y urbano de Chile, con la finalidad de proponer correcciones mediante diversas iniciativas de política pública en esta materia.

Una de las dimensiones en que se observa con más claridad la inequidad del sistema escolar chileno se remite a los resultados de aprendizaje de los estudiantes rurales en comparación con los urbanos. La evaluación de Pisa 2009 dio cuenta de una diferencia que favorece en lectura casi en 50 puntos a los estudiantes de medios urbanos, aún controlando estos resultados por nivel socioeconómico. Pese a la relevancia de considerar esta problemática para asegurar en forma efectiva el derecho a la educación de calidad - más allá de los actuales niveles de cobertura -, no existen hasta el momento antecedentes concluyentes sobre los principales factores que se asocian a las diferencias en este ámbito, ni tampoco sobre su impacto en la continuidad de estudios o en el ingreso al mundo laboral de los jóvenes del sector rural chileno.

El Programa para la Evaluación Internacional de Estudiantes (Pisa) es responsabilidad de la Organización para la Cooperación y el Desarrollo Económico (OCDE), que aplica pruebas estandarizadas para medir competencias en tres ámbitos: Lectura, Matemática y Ciencias. Las pruebas Pisa se aplican desde el año 2000 y Chile ha participado en todas las mediciones, a excepción del año 2003 (en que se aplicó TIMMS). Pisa evalúa el nivel de alfabetización en Lectura, Matemática y Ciencias, como medida de la competencia de los estudiantes de 15 años (sin referencia al curso o nivel que estudian), no obstante la medición del año 2009 se concentró en los temas de lectura.

El trabajo apunta a generar evidencia a partir de los datos Pisa 2009 - empleando un análisis multinivel -, con la finalidad de que los resultados alcanzados contribuyan al diseño de políticas educativas dirigidas al mundo rural, claramente orientadas a disminuir la inequidad de resultados, problemática que no está presente en todos los países evaluados por Pisa, dadas las particularidades de cada contexto (OCDE, 2010). 
Es en esta líneaes destacables señalar que la educación rural no forma parte relevante del discurso actual referente al mejoramiento de las políticas educativas en Chile, sino más bien como un efecto agregado de la política general de educación. En los años noventa la política educativa se enfocó - principalmente - a la expansión de la cobertura y al mejoramiento de la infraestructura y equipamiento escolar, mediante diversos programas de apoyo a la labor educativa en el aula y en el establecimiento escolar, cuyo principal objetivo era mejorar los resultados escolares. ${ }^{1}$ No obstante los esfuerzos en este campo, aún quedan muchos desafíos pendientes, los que implican al 12.9\% de la población del país que habita en el medio rural (INE, 2012). En esta dimensión, Chile ocupa los primeros lugares en términos de acceso y asistencia en educación rural primaria y secundaria en el contexto Latinoamericano (LÓPEZ; PEREYRA; SOURROUILLE, 2007), no obstante lo cual, la brecha por mejorar las condiciones de educabilidad de esta población es significativa, pues las diferencias entre los resultados educacionales de las zonas rurales y urbanas en el país (Chile) indican una contrastede magnitud que no ha sido suficientemente investigado en el ámbito académico, como tampoco se sabe del todo sobre que variables pueden incidir directamente para producir una inflexión importante sobre los resultados en la actualidad, más allá de lo alcanzado en las ultimas dos décadas, que esencialmente ha sido el producto de las políticas nacionales en educación más que de una propuesta integral direccionada en este ámbito. ${ }^{2}$

El propósito del trabajo es identificar, mediante la aplicación de un modelo jerárquico multinivel, aquellos factores que pueden contribuir a explicar las diferencias de resultados en la prueba Pisa de estudiantes del área urbana y rural en el sector de lenguaje. El uso de este procedimiento de análisis estadístico es propicio para indagar en esta temática, pues descompone (desentrama) la proporción de la varianza de los logros promedios de los establecimientos educacionales de ambos tipos, a partir no sólo de su alumnado, sino que también de sus características institucionales y su contexto social.

\section{Antecedentes sobre el estudio y la metodología}

En el plano internacional existe evidencia acerca de las brechas en los resultados educativos entre zonas rurales y urbanas. Al respecto en los países de la OECD (2010) destacan las diferencias de puntajes en Pisa entre ambos sectores, obteniendo los urbanos un mejor desempeño, en países como Turquía, México, Italia y Chile entre otros -, inclusive habiendo controlado por nivel socioeconómico los resultados. Situación que no se manifiesta en todos los países, ejemplo de ello son: Bélgica, Finlandia, Alemania, Irlanda, Grecia, Suiza, Israel, Holanda y el Reino Unido, que no acusan diferencias estadísticamente significativas entre ambos sectores. 
Entre los factores o variables que contribuyen a la explicación de esta situación, Cartwright y Allen (2002) señalan, a partir de una investigación desarrollada en Canadá, que el uso de tecnologías y el acceso a bienes de consumo cultural constituyen factores a considerar para las diferencias de puntajes entre las zonas rurales y urbanas. Asimismo, Alspaugh y Harting (1995) apuntan a considerar las características propias de cada contexto, planteando que para Estados Unidos las diferencias educativas entre las zonas rurales y urbanas pueden ser explicadas por la situación socioeconómica y el aislamiento de las localidades, que inciden en un menor involucramiento y expectativas educativas en la comunidad.

La evidencia empírica para el caso de Chile es insuficiente, de ahí la importancia y necesidad de generar estudios que den cuenta de estas discrepancias, a modo de contribuir a resolver las inequidades educacionales entre el sector rural y urbano, que inciden en el desarrollo del país, debido al alto nivel de centralización en las políticas de desarrollo orientadas a este sector en la sociedad chilena (OYARZÚN; MIRANDA, 2011). En efecto, a la fecha, la población rural sigue presentando menores índices de acceso al derecho a una educación de calidad, con pertinencia y participación. El promedio de escolaridad de la población rural, pese a aumentos sistemáticos descritos, continúa expresando una baja calidad de la educación y mostrando también una profunda desigualdad respecto de los promedios urbanos (INE, 2012).

Previsiblemente, y en base a estudios ya clásicos en el ámbito educacional, Coleman (1966) en los Estados Unidos y Bourdieu y Passeron (1970) en Europa, observamos que el sistema educativo ha tendido a reproducir la estructura social y sus desigualdades paralelas. Respecto a las desigualdades socioeconómicas, Espíndola y León (2002) sostienen que los bajos ingresos de los estudiantes de estratos más pobres son decisivos para el retraso y abandono escolar, y que estas disparidades en las tasas de deserción escolar contribuyen a la reproducción de desigualdades sociales. Esta relación entre la institución escolar y la población atendida tiene efectos en el nivel de eficiencia del sistema, esto es, en su capacidad de garantizar el derecho a la educación, en le caso chileno la incidencia de las variables estructurales asociadas a los factores socioeconómicos es determinante (HSIEH; URQUIOLA, 2002, 2006; TREVIÑO; DONOSO, 2010; MIZALA; TORCHE, 2012).

No obstante lo anterior, especialistas sostienen que en nuestro medio los aspectos referidos a la gestión y efectividad de las escuelas son más importantes que otras iniciativas, algunas de ellas tan costosas como la ampliación de la jornada escolar completa (PAREDES; PAREDES, 2009) y que los problemas se deben a que la autonomía de los establecimientos, pero ha sido acompañada por la responsabilización por los resultados (BEYER; VELASCO, 2010). Relevando de este modo la importancia del "efecto escuela" en el nivel de logro de los estudiantes. No obstante los resultados del país en distintas series de tiempo, confirman el peso de los factores 
Inequidades invisibles en la educación Chilena...

socioeconómicos y territoriales por sobre los factores intraescuela (DRAGO; PAREDES, 2011; MIZALA; TORCHE, 2012; DONOSO; ARIAS, 2013).

Durante la última década se ha masificado el uso de la técnica estadística de modelos jerárquico multinivel en la investigación educativa, en particular cuando se trata de estudios que analizan datos jerárquicamente relacionados (CERVINI, 2005), que en este caso corresponde a: estudiantes con características individuales y familiares que pertenecen a establecimientos educacionales con determinadas características contextuales. Esta metodología se adapta a los objetivos del trabajo de mejor manera que los modelos tradicionales de regresión, puesto el principio de independencia de los residuos no es respetado en datos con estructura jerárquica, donde la observación individual no es totalmente independiente (CERVINI, 2005; GOLDSTEIN; SAMMO, 1997), dicho de otro modo, cualquier sujeto - estudiante - que pertenezca a un mismo grupo - establecimiento educacional - comparte un conjunto de estímulos que favorece la homogeneidad (CASTRO; GAVIRIA, 2005).

En este estudio se ha definido dos niveles: alumnos y establecimientos educacionales. El efecto aula, dadas las características de la muestra, no podrá ser observado, puesto el sistema de registro de datos de Pisa no proporciona los datos suficientes para incluir este nivel en el modelo, lo que podría generar una sobreestimación de la varianza en ambos niveles. ${ }^{3}$

En razón de ello, el objetivo general del estudio es determinar las características individuales, familiares, de la escuela y de la experiencia escolar de los estudiantes chilenos que rindieron la prueba Pisa 2009, que inciden en la brecha de resultados en lectura entre estudiantes de zonas rurales y urbanas.

Por tanto, el primer objetivo específico es analizar si los factores individuales, familiares, de la escuela y de la experiencia escolar de los estudiantes se relacionan con las brechas de resultados en lectura para Pisa 2009 según su localización. Este objetivo se desglosa en las siguientes preguntas de investigación: ¿En qué medida las diferencias de rendimiento son atribuibles alas características individuales del estudiante y su familia y/o a la composición del establecimiento educacional? ¿Qué relevancia posee en el caso chileno la localización del establecimiento educacional para el rendimiento en lenguaje de los estudiantes en Pisa?

Como se indicó, el Pisa aplica pruebas estandarizadas para medir competencias en tres ámbitos: Lectura, Matemática y Ciencias. En cada ciclo de medición, Pisa establece una de las tres áreas como la principal, por lo que se incluyen sustantivamente más preguntas de dicha área que de las demás, con el objetivo de reportarla con mayor profundidad y riqueza.

Los resultados de Pisa 2009 confirmaron las noticias positivas para el país desde el año 2006, evidenciando una mejoría en los aprendizajes alcanzados en el área de 
Lectura por los estudiantes de 15 años. Sin embargo, los resultados de los estudiantes chilenos están sobre el promedio de los países latinoamericanos, pero siguen bajo el promedio de los países de la OCDE.

\section{Datos}

Se realizó un análisis multivariado con datos provenientes de las pruebas de lenguaje del OECD - Programme for International Student Assessment (Pisa) - aplicadas el año 2009 a estudiantes de escuelas de Chile. La muestra total correspondió a 5.669 estudiantes pertenecientes a 200 escuelas participantes en la medición. El total de casos ha sido dividido según área de residencia de los estudiantes, seccionándose en dos categorías: rural y no rural. Los datos utilizados fueron ponderados de acuerdo a las proyecciones de porcentaje de población desarrolladas por el INE (2010).

\section{Variables}

La variable dependiente corresponde a los puntajes obtenidos por los alumnos en las pruebas estandarizadas de lenguaje Pisa. Las variables independientes son: (a) características individuales del estudiante y su familia, y (b) composición de la escuela

Respecto de la primera variable independiente, Características individuales del alumno, los valores considerados fueron:

- Género

- Asistencia a Educación Preescolar

- Repitencia

- Composición familiar

- Índice de bienes materiales

- Cantidad de libros en el hogar

- Relación con sus profesores

- Comportamiento alumnos en clases

- Uso TICs en el hogar

- Nivel Escolaridad Madre
Femenino (1) - Masculino (0)

Asistió (1) - No asistió (0)

No repitió (1) - Repitió (0)

Vive con la madre (1) - No vive con la madre (0)

De un total de 8 bienes durables y de uso en el hogar, se calcula un índice de razón con 8 atributos

Suficiente (1) - Insuficiente (0).

Buena (1) - No muy buena (0).

Adecuado (1) - Inadecuado (0)

De un total de 9 bienes durables y de uso en el hogar, se calcula un índice de razón con 9 atributos

De un total de 3 categorías, se calcula un índice de 3 atributos 
Inequidades invisibles en la educación Chilena...

- Nivel Escolaridad Padre

- Gasto de la familia en Escolaridad

- Hábito de lectura de los padres
De un total de 3 categorías, se calcula un índice de 3 atributos

Índice de razón que contempla 6 atributos de gasto en Pesos Chilenos

Adecuado (1) - Inadecuado (0)

En tanto para la segunda variable independiente, Composición de la escuela, los valores fueron:

- Dependencia establecimiento

- Zona

- Presión de los padres al Colegio

- Selección de alumnos por rendimiento

- Uso TICs en el Colegio
No Pública (1) - Pública (0)

Rural (1) - No Rural (0)

Presión constante (1) - Presión escasa (0)

Hay selección (1) - No hay selección (0)

De un total de 6 bienes durables y de uso en el colegio, se calcula un índice de razón con 6 atributos

\section{Técnica y proceso de análisis}

Para el análisis de las relaciones entre el rendimiento escolar promedio en la prueba de lenguaje y las diferentes variables que explicarían este fenómeno, se utilizarán los programas SPSS y HML. Los datos permitieron definir modelos con 2 niveles: alumnos (nivel 1), establecimiento educacional (nivel 2). Como bien se explicó, fue imposible incluir un tercer nivel de análisis dadas las características de la información disponible.

Tabla I

Variables descriptivas

\begin{tabular}{|c|c|c|c|c|c|}
\hline Nivel & Variable & Categoría & $\%$ & Media & Desv. Est. \\
\hline \multirow[t]{6}{*}{ Nivel 1} & Género & Femenino & 49 & $\mathrm{n} / \mathrm{a}$ & $\mathrm{n} / \mathrm{a}$ \\
\hline & & & & & \\
\hline & Asistencia Pre-escolar & $\begin{array}{c}\text { Asiste } \\
\text { No asiste }\end{array}$ & $\begin{array}{l}85 \\
15\end{array}$ & $\mathrm{n} / \mathrm{a}$ & $\mathrm{n} / \mathrm{a}$ \\
\hline & Repitencia & $\begin{array}{c}\text { Repite } \\
\text { No repite }\end{array}$ & $\begin{array}{l}28 \\
72\end{array}$ & $\mathrm{n} / \mathrm{a}$ & $\mathrm{n} / \mathrm{a}$ \\
\hline & Composición Familiar & $\begin{array}{c}\text { Vive con la madre } \\
\text { No vive con la madre }\end{array}$ & $\begin{array}{c}91 \\
9\end{array}$ & $\mathrm{n} / \mathrm{a}$ & $\mathrm{n} / \mathrm{a}$ \\
\hline & Índice Bienes Materiales & $\begin{array}{c}1 \text { bien } \\
2 \text { bienes }\end{array}$ & $\begin{array}{l}0.1 \\
0.7\end{array}$ & & \\
\hline
\end{tabular}




\begin{tabular}{|c|c|c|c|c|}
\hline & $\begin{array}{l}3 \text { bienes } \\
4 \text { bienes } \\
5 \text { bienes } \\
6 \text { bienes } \\
7 \text { bienes } \\
8 \text { bienes }\end{array}$ & $\begin{array}{l}3.4 \\
7.5 \\
13.8 \\
18.7 \\
23.4 \\
32.2\end{array}$ & 5.5 & 1.5 \\
\hline $\begin{array}{l}\text { Cantidad Libros en } \\
\text { Hogar }\end{array}$ & $\begin{array}{l}\text { Suficiente } \\
\text { Insuficiente }\end{array}$ & $\begin{array}{l}19 \\
81\end{array}$ & $\mathrm{n} / \mathrm{a}$ & $\mathrm{n} / \mathrm{a}$ \\
\hline Relación Profesores & $\begin{array}{c}\text { Buena } \\
\text { No muy buena }\end{array}$ & $\begin{array}{l}67 \\
33\end{array}$ & $\mathrm{n} / \mathrm{a}$ & $\mathrm{n} / \mathrm{a}$ \\
\hline $\begin{array}{l}\text { Comportamiento } \\
\text { alumnos en clases }\end{array}$ & $\begin{array}{l}\text { Adecuado } \\
\text { Inadecuado }\end{array}$ & $\begin{array}{l}16.5 \\
83.5\end{array}$ & $\mathrm{n} / \mathrm{a}$ & $\mathrm{n} / \mathrm{a}$ \\
\hline Uso TICs Hogar & $\begin{array}{c}1 \text { bien } \\
2 \text { bienes } \\
3 \text { bienes } \\
4 \text { bienes } \\
5 \text { bienes } \\
6 \text { bienes } \\
7 \text { bienes } \\
8 \text { bienes } \\
9 \text { bienes }\end{array}$ & \begin{tabular}{c|c|}
3.6 & \\
7.8 \\
9.3 \\
12.1 \\
12.4 \\
16.6 \\
18.3 \\
14.2 \\
5.7
\end{tabular} & 4.5 & 2.1 \\
\hline Escolaridad Madre & $\begin{array}{l}\text { Básica incompleta/ } \\
\text { completa } \\
\text { Media incompleta/ } \\
\text { completa } \\
\text { Educación Superior }\end{array}$ & $\begin{array}{l}36.2 \\
36.2 \\
27.7\end{array}$ & 2 & 0.8 \\
\hline Escolaridad Padre & $\begin{array}{l}\text { Básica incompleta/ } \\
\text { completa } \\
\text { Media incompleta/ } \\
\text { completa } \\
\text { Educación Superior }\end{array}$ & $\begin{array}{l}35.2 \\
33.1 \\
31.7\end{array}$ & 2 & 0.8 \\
\hline $\begin{array}{l}\text { Gasto Familia } \\
\text { en escolaridad }\end{array}$ & $\begin{array}{c}\text { No gasta } \\
\$ 1-\$ 42.000 \\
\$ 42.001-\$ 168.000 \\
\$ 168.001-\$ 294.000 \\
\$ 294.001-\$ 426.000 \\
\text { Más de } \$ 426.001\end{array}$ & $\begin{array}{r}10.8 \\
30.2 \\
46.8 \\
5.0 \\
1.6 \\
5.6\end{array}$ & 1.7 & 1.1 \\
\hline Hábito Lectura Padres & $\begin{array}{c}\text { Adecuado } \\
\text { Inadecuado }\end{array}$ & $\begin{array}{l}27 \\
73\end{array}$ & $\mathrm{n} / \mathrm{a}$ & $\mathrm{n} / \mathrm{a}$ \\
\hline
\end{tabular}


Inequidades invisibles en la educación Chilena...

\begin{tabular}{|c|c|c|c|c|c|}
\hline Nivel & Variable & Categoría & $\%$ & Media & Desv. Est. \\
\hline \multirow[t]{5}{*}{ Nivel 2} & Dependencia & $\begin{array}{l}\text { Pública } \\
\text { No Pública }\end{array}$ & $\begin{array}{l}44 \\
56\end{array}$ & $\mathrm{n} / \mathrm{a}$ & $\mathrm{n} / \mathrm{a}$ \\
\hline & Zona & $\begin{array}{c}\text { Rural } \\
\text { No Rural }\end{array}$ & $\begin{array}{c}6 \\
94\end{array}$ & $\mathrm{n} / \mathrm{a}$ & $\mathrm{n} / \mathrm{a}$ \\
\hline & Presión Padres & $\begin{array}{l}\text { Presión Constante } \\
\text { Presión escasa }\end{array}$ & $\begin{array}{l}22 \\
78\end{array}$ & $\mathrm{n} / \mathrm{a}$ & $\mathrm{n} / \mathrm{a}$ \\
\hline & $\begin{array}{l}\text { Selección alumnos por } \\
\text { rendimiento }\end{array}$ & $\begin{array}{l}\text { Con selección } \\
\text { Sin selección }\end{array}$ & $\begin{array}{l}31 \\
69\end{array}$ & $\mathrm{n} / \mathrm{a}$ & $\mathrm{n} / \mathrm{a}$ \\
\hline & Uso TICs Hogar & $\begin{array}{c}1 \text { bien } \\
2 \text { bienes } \\
3 \text { bienes } \\
4 \text { bienes } \\
5 \text { bienes } \\
\text { 6bienes }\end{array}$ & $\begin{array}{c}17.5 \\
36.2 \\
41.8 \\
4.5 \\
0 \\
0.5\end{array}$ & 1.8 & 0.8 \\
\hline
\end{tabular}

\section{Modelos y Resultados}

Se emplearon procedimentalmente cuatro modelos de análisis: primero el vacío, luego dos modelos parciales y finalmente el modelo total. Su detalle es el siguiente:

a) Modelos vacíos: técnica de análisis de la varianza de la variable lenguaje, sin predictor. Se procesan 2 modelos multinivel para evaluar los efectos de las características individuales del alumno, la composición de las escuelas y las características de la región, sin control.

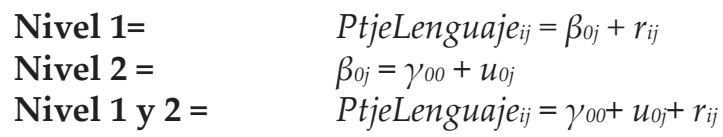

Con un Sigma $\left(\sigma^{2}\right)$ de 3264.66481, un Tao de 2947.00806 y un $86 \%$ de la varianza observada de la gran media no correspondiente al error muestral, es posible decir que un $47 \%$ de la varianza total es explicada por las características de la escuela. Por tal motivo se establece una mayor importancia de variables de Nivel 1 para explicar los puntajes en lenguaje Pisa en los estudiantes de nuestro país.

b) Modelo Nivel 1: Factores individuales del alumno y su familia que predicen el rendimiento de lenguaje de los alumnos que rindieron la prueba Pisa 2009

Nivel 1= PtjeLenguaje $e_{i j}=\beta_{0 j}+\beta_{1 j}^{*}\left(\right.$ Sexo $\left._{i j}\right)+\beta_{2 j}{ }^{*}\left(\right.$ Educación Preescolar $\left._{i j}\right)+$ $\beta_{3 j}^{*}\left(\right.$ Repetición $\left._{i j}\right)+\beta_{4 j}^{*}\left(\right.$ Vive Madre $\left._{i j}\right)+\beta_{5 j}^{*}$ (Índice Bienes Culturales $i j)+\beta_{6 j}{ }^{*}\left(\right.$ Cantidad Libros $\left._{i j}\right)+\beta_{7 j}^{*}\left(\right.$ Relación Profesores $\left._{i j}\right)$ 
$+\beta_{8 j}^{*}($ Comportamiento Alumnos $\left.i j)\right)+\beta_{9 j}^{*}\left(\right.$ Tics Hogar $\left._{i j}\right)+\beta_{10 j}^{*}($ Uso Computador $i j)+\beta_{11 j^{*}}($ Escolaridad Madre $i j)+\beta_{12 j}{ }^{*}$ (Escolaridad Padre $\left._{i j}\right)+\beta_{13 j}^{*}\left(\right.$ Gasto Educación $\left._{i j}\right)+\beta_{14 j}^{*}($ Hábito Lectura Padres $\left._{i j}\right)+r_{i j}$

Con un Sigma $\left(\sigma^{2}\right)$ de 2712.26890 , un Tao de 688.43050 y un $71 \%$ de la varianza observada de la gran media no correspondiente al error muestral, un $18 \%$ de la varianza intragrupo es explicada por las variables contrastadas en el Modelo de Nivel 1.

c) Modelo Nivel 2: Composición establecimiento educacional

Nivel $2=\quad \boldsymbol{\beta}_{0 j}=\gamma_{00}+\gamma_{01}{ }^{*}($ Dependencia $)+\gamma_{02}{ }^{*}($ Zona $)+\gamma_{03}{ }^{*}$ (Presión Padres Colegio $)+\gamma_{04}^{*}($ Selección Alumnos $)+\gamma_{05}^{*}($ Tics Colegio $j)+u_{0 j}$

Con un Sigma $\left(\sigma^{2}\right)$ de2687.81398, un Tao de 449.38177 y un $64 \%$ de la varianza observada de la gran media no correspondiente al error muestral, un $85 \%$ de la varianza intragrupo es explicada por las variables contrastadas en el Modelo de Nivel 2.

d) Modelo final $=$ PtjeLenguaje $e_{i j}=\gamma_{00} \gamma_{01}{ }^{*}$ Dependencia $+\gamma_{02}{ }^{*}$ Zona $+\gamma_{03}{ }^{*}$ Presión Padres Colegio $+\gamma_{04}{ }^{*}$ Selección Alumnos ${ }_{j}+\gamma_{05}{ }^{*}$ Tics Colegio ${ }_{j}+\gamma_{10}{ }^{*} \operatorname{Sexo}_{i j}$ $+\gamma_{20}{ }^{*}$ EducaciónPreescolar ${ }_{i j}+\gamma_{30}{ }^{*}$ Repetición $_{i j}+\gamma_{40}{ }^{*}$ Vive Madre $_{i j}$

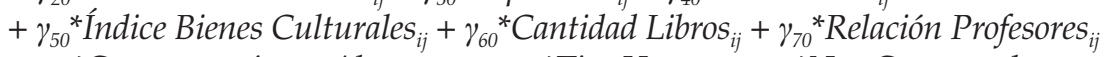
$+\gamma_{80}{ }^{*}$ Comportamiento Alumnos $i j+\gamma_{90}{ }^{*}$ Tics Hogar $_{i j}+\gamma_{100}{ }^{*}$ Uso Computador $_{i j}$

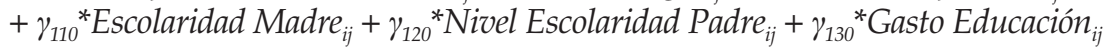
$+\gamma_{140}{ }^{*}$ Hábito Lectura Padres $i j+u_{0 j}+r_{i j}$

La variable predictiva de mayor significación y relevancia a nivel 2 de escuela, es la zona o área territorial donde se localiza el establecimiento educacional (sig. 0.001). Los establecimientos rurales provocarán que sus estudiantes obtengan 44.316922 puntos menos con respecto a la gran media de puntajes de Lenguaje Pisa. Por el contrario, aquellos de zonas no rurales (ciudad) obtendrán 44.316922 puntos más respecto a la gran media de puntajes.

La segunda variable es la presión que ejercen los padres en el colegio (sig. 0.05). Se vislumbra que los establecimientos que reciben presión de los apoderados, sus alumnos obtienen 21.928044 puntos más que la gran media de puntajes de Lenguaje Pisa. En el caso de no recibir presión constante o no recibir presión, los alumnos obtendrán 21.928044 puntos menos en promedio.

La tercera variable en el caso del Nivel 2 es la selección de estudiantes, bajo el criterio del rendimiento académico que ejercen los colegios (sig. 0.05). Al amparo de este fenómeno, los alumnos obtienen 21.274203 puntos más que la gran media de puntajes de Lenguaje Pisa. Al contrario, en los que no seleccionan sus alumnos registran 21.274203 puntos menos en promedio. 
Inequidades invisibles en la educación Chilena...

\section{Tabla II}

Modelo Final Nivel 1 y 2

\begin{tabular}{|c|c|c|c|}
\hline NIVEL 2 & $\begin{array}{l}\text { Coeficiente } \\
\\
313.680255\end{array}$ & $\begin{array}{c}\begin{array}{c}\text { Error } \\
\text { estándar }\end{array} \\
13.045989\end{array}$ & $\begin{array}{r}p \text {-value } \\
\\
<0.001\end{array}$ \\
\hline $\begin{array}{l}\text { Dependencia no Pública } \\
\text { Dependencia Pública }\end{array}$ & $\begin{array}{r}13.361241 \\
-13.361241\end{array}$ & $\begin{array}{r}7.102585 \\
*\end{array}$ & $\begin{array}{r}0.065 \\
*\end{array}$ \\
\hline $\begin{array}{l}\text { Zona Rural } \\
\text { Zona no Rural }\end{array}$ & $\begin{array}{r}-44.316922 \\
44.316922\end{array}$ & $\begin{array}{r}13.158988 \\
* * *\end{array}$ & $\begin{array}{r}<0.001 \\
* * *\end{array}$ \\
\hline $\begin{array}{l}\text { Presión de Padres al Colegio } \\
\text { Sin Presión de Padres al Colegio }\end{array}$ & $\begin{array}{r}21.928044 \\
-21.928044\end{array}$ & $\begin{array}{r}8.455338 \\
* *\end{array}$ & $\begin{array}{r}0.012 \\
* *\end{array}$ \\
\hline $\begin{array}{l}\text { Selección de Alumnos } \\
\text { Sin Selección de Alumnos }\end{array}$ & $\begin{array}{r}21.274203 \\
-21.274203 \\
\end{array}$ & $\begin{array}{r}10.650069 \\
* *\end{array}$ & $\begin{array}{r}0.050 \\
* *\end{array}$ \\
\hline Uso TICs Colegios & -4.021439 & 5.146004 & 0.438 \\
\hline $\begin{array}{l}\text { NIVEL } 1 \\
\text { Sexo Femenino } \\
\text { Sexo Masculino }\end{array}$ & $\begin{array}{r}9.659271 \\
-9.659271\end{array}$ & $\begin{array}{r}3.424189 \\
* *\end{array}$ & $\begin{array}{r}0.005 \\
* *\end{array}$ \\
\hline $\begin{array}{l}\text { Asistencia a Educación Preescolar } \\
\text { Inasistencia a Educación Preescolar }\end{array}$ & $\begin{array}{r}13.019944 \\
-13.019944 \\
\end{array}$ & $\begin{array}{r}3.094379 \\
* * * \\
\end{array}$ & $\begin{array}{r}<0.001 \\
* * *\end{array}$ \\
\hline $\begin{array}{l}\text { Sin repetición de cursos } \\
\text { Con repetición de cursos }\end{array}$ & $\begin{array}{r}46.928977 \\
-46.928977\end{array}$ & $\begin{array}{r}4.533174 \\
* * *\end{array}$ & $\begin{array}{r}<0.001 \\
* * *\end{array}$ \\
\hline $\begin{array}{l}\text { Vive con la madre } \\
\text { No vive con la madre }\end{array}$ & $\begin{array}{r}38.716828 \\
-38.716828\end{array}$ & $\begin{array}{r}6.474977 \\
* * *\end{array}$ & $\begin{array}{r}<0.001 \\
* * *\end{array}$ \\
\hline Índice de Bienes Culturales & 3.312520 & 1.317494 & 0.012 \\
\hline $\begin{array}{l}\text { Cantidad de libros Suficientes } \\
\text { Cantidad de libros Insuficientes }\end{array}$ & $\begin{array}{r}15.625149 \\
-15.625149\end{array}$ & $\begin{array}{r}5.340267 \\
* *\end{array}$ & $\begin{array}{r}0.004 \\
* *\end{array}$ \\
\hline $\begin{array}{l}\text { Relación con profesores adecuada } \\
\text { Relación con profesores inadecuada }\end{array}$ & $\begin{array}{r}7.305440 \\
-7.305440\end{array}$ & $\begin{array}{r}4.255771 \\
*\end{array}$ & $\begin{array}{r}0.086 \\
*\end{array}$ \\
\hline $\begin{array}{l}\text { Comportamiento Alumnos en Clases adecuado } \\
\text { Comportamiento Alumnos en Clases inadecuado }\end{array}$ & $\begin{array}{r}0.984507 \\
-0.984507\end{array}$ & 4.808136 & 0.838 \\
\hline Uso TICs en el Hogar & -1.041561 & 1.132130 & 0.358 \\
\hline $\begin{array}{l}\text { Uso del computador } \\
\text { No uso del computador }\end{array}$ & $\begin{array}{r}28.013732 \\
-28.013732\end{array}$ & $\begin{array}{r}11.488091 \\
* *\end{array}$ & $\begin{array}{r}0.015 \\
* *\end{array}$ \\
\hline Nivel Escolaridad Madre & 5.182181 & 3.187263 & 0.100 \\
\hline Nivel Escolaridad Padre & 2.873864 & 2.832303 & 0.311 \\
\hline Gasto en Escolaridad & 2.293994 & 2.101708 & 0.275 \\
\hline $\begin{array}{l}\text { Hábito de Lectura de los Padres adecuado } \\
\text { Hábito de Lectura de los Padres inadecuado }\end{array}$ & $\begin{array}{r}0.518381 \\
-0.518381\end{array}$ & 3.759896 & 0.890 \\
\hline
\end{tabular}


La cuarta variable es la dependencia educacional de los establecimientos escolares (sig. 0.1). Se observa claramente que aquellas escuelas con dependencia privada conseguirán que sus alumnos obtengan 13.361241 puntos más respecto a la gran medio de puntajes de Lenguaje Pisa. En el lado opuesto, si la dependencia es pública, los alumnos obtendrán en promedio 13.361241 puntos menos respecto de la gran media.

Con referencia a las variables concernientes con los alumnos y sus familias, la primera categoría a evaluar es la asistencia a la educación preescolar por los estudiantes (sig. 0.001). Efectivamente los que han asistido obtendrán 13.019944 puntos más respecto a la gran media de puntajes. Al contrario, los que no asistieron obtendrán 13.019944 puntos menos en promedio.

La repetición de cursos (sig. 0.001) también es una variable a considerar. No haber repetido curso permite al alumno sumar 46.928977 puntos en promedio respecto a la gran media de puntajes Pisa lenguaje. Al contrario, los estudiantes que si lo han hecho repetido obtendrán los mismos puntos menos.

Respecto al apartado de composición familiar de los estudiantes, vivir con la madre es una variable a estimar (sig. 0.001). En este sentido, la presencia materna en el hogar permite agregar 38.716828 puntos más respecto de la gran media de puntajes. Al contrario, el que el estudiante no vive con su madre, resta la misma cantidad de puntos en promedio en relación con la gran media.

A su vez, el género (sig. 0.05) es una variable que produce gran diferencia. El alumno de género masculino, ponderará en promedio 9.659271 puntos menos respecto a la gran media de puntajes y como es de esperar, la mujer ponderará 9.659271 puntos más.

Si consideramos el índice de bienes culturales (sig. 0.05), que evaluaba la posesión de elementos que aportan a la labor de aprendizaje (p.e. tener un lugar donde estudiar en el hogar, computadores, etc.), observamos que cada vez que aumenta la propiedad de una unidad (de un total de ocho atributos) los estudiantes podrán obtener en promedio 3.312520 puntos más respecto a la gran media de puntajes de la prueba Pisa lenguaje 2009.

En relación a la cantidad de libros que poseen en el hogar (sig. 0.05), si la familia tiene más de 101 libros en casa (que es un parámetro de suficiencia para estos elementos) los estudiantes podrán obtener en promedio 15.625149 puntos más respecto a la gran media de puntajes. Por el contrario, si es que la familia posee una cantidad menor de libros, los estudiantes restarán la misma cantidad de puntos en promedio en la prueba de Lenguaje.

Al momento de conocer si acaso los estudiantes han utilizado alguna vez en su vida un computador (sig. 0.05), podemos observar que aquellos estudiantes que 
efectivamente lo han ocupado obtendrán 28.013732 puntos más respecto a la gran media de puntajes, mientras que aquellos que jamás han utilizado un computador ponderarán la misma cantidad de puntos menos en promedio.

Finalmente, otras variables a considerar son la importancia que posee la relación del alumno con sus profesores en la escuela (sig. 0.1) y el nivel de escolaridad de la madre (sig. 0.1). En el primer caso, cuando el alumno posee una buena relación con sus profesores, éste podrá obtener en promedio 7.305440 puntos más en la prueba de lenguaje, mientras que en el caso contrario restará la misma cantidad de puntos. En el caso de la segunda variable, cada vez que la madre avance en un nivel educativo (p.e. de educación básica a educación media) el alumno obtendrá 5.182181 puntos más en la prueba de lenguaje.

De igual modo, es necesario mencionar la existencia de variables que no presentaron un nivel de significación adecuado para el análisis final. En el caso de las variables a nivel escuela el uso de TICs en el establecimiento (sig. .0438). En las variables individuales, las que no calificaron para el análisis final fueron: comportamiento de los alumnos en clases (sig. 0.838), uso de TICs en el hogar (sig. 0.358), nivel de escolaridad del padre (sig. 0.311), gasto en escolaridad que realiza la familia (sig. 0.275) y hábito de lectura de los padres (sig. 0.890).

El debate de estos resultados y la explicación respecto de las consecuencias para el caso de los alumnos residentes en las zonas rurales de Chile en comparación con aquellos que residen en otros lugares se expone en la sección siguiente.

\section{Discusión y conclusiones}

\section{Variables de primer nivel: características del estudiante y su familia}

La primera parte del análisis se dirige a desentramar cuáles son las características del estudiante y de su familia que estarían afectando directamente el rendimiento ponderado Pisa de Lenguaje para quienes estudian en zonas rurales respecto a sus símiles de otras localidades.

\section{Tabla III}

¿Asistió a la educación preescolar? (\%)

\begin{tabular}{|llll|}
\hline & \multicolumn{2}{c}{ Zona/ área } & \\
\cline { 2 - 3 } & Rural & No Rural & Total \\
\cline { 2 - 3 } $\mathrm{No}$ & 29,6 & 12,1 & 15,0 \\
\cline { 2 - 3 } $\mathrm{Si}$ & 70,4 & 87,9 & 85,0 \\
\hline
\end{tabular}


Los estudiantes para obtener en promedio 13.019944 puntos más en su rendimiento deben haber asistido a algún tipo de educación preescolar, los alumnos de zonas rurales se encuentran en desventaja respecto a las demás localidades y a la población en general (Tabla 3). En promedio existe un 15\% de estudiantes en Chile que no cursaron estos estudios. Sin embargo, en zonas rurales este porcentaje duplica ese valor porcentual, llegando al 30\% de la población. Hecho que se traduce en una merma del promedio ponderado de 13.019944 respecto a la media.

\section{Tabla IV}

¿Ha repetido algún curso? (\%)

\begin{tabular}{|cccc|}
\hline & \multicolumn{2}{c}{ Zona/ área } & \\
\cline { 2 - 3 } & Rural & No Rural & Total \\
\cline { 2 - 3 } No & 49.5 & 75,5 & 72,3 \\
\cline { 2 - 3 } Si & 50,5 & 24,5 & 27,7 \\
\hline
\end{tabular}

Otro factor de consideración es la repitencia de curso de los estudiantes. Los datos generales Pisa muestran que un 28\% de los estudiantes han repetido al menos un año durante su educación formal. Sin embargo, en el sector rural algo más de la mitad de los estudiantes $(50,5 \%)$ ha repetido, lo que impacta que en promedio sus puntajes ponderados tengan 46.928977 puntos menos respecto a los otros sectores. De esta forma la repitencia de estudiantes en el medio rural es una variable que gravita significativamente y su corrección podría ser más fácil y con mayor impacto de corto plazo, que la anteriormente expuesta, en tanto se implementaran políticas efectivas de ampliación de la cobertura preescolar dada su positiva incidenciaen la reducción de la repitencia.

Tabla V

¿Vive con su madre? (\%)

\begin{tabular}{|lccc|}
\hline & \multicolumn{2}{c}{ Zona/ área } & \\
\cline { 2 - 3 } & Rural & No rural & Total \\
\cline { 2 - 3 } No & 18,7 & 7,6 & 8,7 \\
\cline { 2 - 3 } Si & 81,3 & 92,4 & 91,3 \\
\hline
\end{tabular}

La tercera variable de interés es la convivencia con la madre, donde la tendencia nacional es afirmativa para el $91 \%$ de los estudiantes. Los porcentajes para las zonas no rurales presentan alta semejanza con ese valor, no obstante en el sector 
Inequidades invisibles en la educación Chilena...

rural hay una diferencia significativa de 10 puntos porcentuales menos (81\%), que señala una proporción mayor de estudiantes que no pueden obtener en promedio 38.716828 más en la prueba Pisa por este factor. Una hipótesis posible para explicar esta situación, se refiere a los problemas de cobertura, la proporción de estudiantes que debe emigrar al iniciar la secundaria, la dispersión territorial de la población y la migración de los padres.

\section{Tabla VI}

Cantidad de libros en el hogar (\%)

\begin{tabular}{|lccc|}
\hline & \multicolumn{2}{c}{ Zona/ área } & \\
\cline { 2 - 3 } & Rural & No Rural & Total \\
Insuficiente & 97,6 & 78,1 & 81,1 \\
\cline { 2 - 3 } Suficiente & 2,4 & 21,9 & 18,9 \\
\hline
\end{tabular}

La revisión de la cantidad de libros que poseen los hogares muestra ciertas diferencias, entendiendo que en lo grueso es claramente insuficiente pues un $81 \%$ de los hogares no posee una cifra de libros adecuada (más de 100 ejemplares). No obstante, en las zonas rurales, esta insuficiencia es más pronunciada puesto que un $98 \%$ de los alumnos que rindieron la prueba Pisa no poseen suficientes libros en sus casas, lo que se traduce en promedio en una resta de 15.6625149 puntos respecto a la gran media de puntajes.

\section{Tabla VII}

Género de los estudiantes (\%)

\begin{tabular}{|lccc|}
\hline & \multicolumn{2}{c}{ Zona/ área } & \\
\cline { 2 - 3 } & Rural & No Rural & Total \\
Masc. & 86,0 & 50,5 & 50,1 \\
\cline { 2 - 3 } Fem & 14,0 & 49,5 & 49.9 \\
\hline
\end{tabular}

El género de los estudiantes es una variable que produce gran diferencia entre la zona rural versus otros lugares. En este sentido, el promedio nacional nos dice que el 50,1\% es hombre, manteniéndose la tendencia en zonas no rurales. Pero, en el caso de la muestra rural, se observa un salto de casi 40 puntos porcentuales (86\%), lo que merma el rendimiento promedio de los estudiantes de aquel sector. Se constata una diferencia que deberá verse si su ocurrencia marca una tendencia. 
Tabla VIII

Valorización de la relación de los estudiantes con el profesor (\%)

\begin{tabular}{|lccc|}
\hline & \multicolumn{2}{c|}{ Zona/ área } & \\
\cline { 2 - 3 } & Rural & No Rural & Total \\
\cline { 2 - 3 } No muy buena & 19,1 & 33,5 & 33.1 \\
\cline { 2 - 3 } Buena & 80,9 & 66,5 & 66,9 \\
\hline
\end{tabular}

Del conjunto de variables consideradas, la única que tiene un comportamiento diferente a lo expuesto es el tipo de relación de los alumnos con sus profesores. Al respecto, la tendencia nacional dice que un $67 \%$ de los alumnos tiene buena relación con sus profesores, pero para el caso de los sectores rurales, se registra una diferencia positiva de casi 15 puntos porcentuales (81\%), lo que se traduce en un aumento significativo de 7.305440 respecto al promedio ponderado de la prueba Pisa. Esta variable es un factor relevante a potenciar en el medio rural, y a corregir en el urbano.

\section{Tabla IX}

Posesión de Bienes culturales

\begin{tabular}{|c|c|c|c|}
\hline & \multicolumn{2}{|c|}{ Zona/ área } & \multirow[b]{2}{*}{ Total } \\
\hline & Rural & No Rural & \\
\hline 1 bien & 1,3 & 0,1 & 0,2 \\
\hline 2 bienes & 0,0 & 0,5 & 0,7 \\
\hline 3 bienes & 6,7 & 2,3 & 3,4 \\
\hline 4 bienes & 16,8 & 5,5 & 7,4 \\
\hline 5 bienes & 35,9 & 12.0 & 13,9 \\
\hline 6 bienes & 20,2 & 18,2 & 18,8 \\
\hline 7 bienes & 12,2 & 23.9 & 23,6 \\
\hline 8 bienes & 6,9 & 37,5 & 32,1 \\
\hline
\end{tabular}

Para las variables de primer nivel que evalúan las características individuales de los estudiantes y de sus familias, se ha generado el índice de bienes culturales. Cada vez que aumenta un bien en el hogar, el estudiante aumentará en promedio 3.312520 puntos en su puntaje Pisa. No obstante, manteniendo la tendencia general, para el caso de las zonas rurales hay evidencia de menor posesión de bienes culturales en las familias respecto a otros lugares. Por ejemplo, los promedios totales dicen que 
un $32 \%$ de los hogares posee 7 bienes culturales (lo que haría que los estudiantes sumaran en promedio 23.18764), pero para los sectores rurales sólo un 6,9\% de sus estudiantes poseen dicha cantidad de bienes en sus hogares, incidiendo en sus oportunidades educacionales. De esta forma la política educacional se enfrenta a un desafío relevante: aumentar el acceso a bienes culturales en el medio rural puede tener un rápido impacto valioso, adicionalmente generaría otras externalidades positivas, pues reduce en algo la dependencia de los resultados del accionar del profesor, lo que permite mayor estabilidad de los logros.

\section{Tabla X}

Nivel de escolaridad de la madre

\begin{tabular}{|c|c|c|c|}
\hline & \multicolumn{2}{|c|}{ Zona/ área } & \multirow[b]{2}{*}{ Total } \\
\hline & Rural & No Rural & \\
\hline Básica completa o Incomp. & 75,5 & 28,5 & 35,9 \\
\hline Media Completa o Incomp. & 19,8 & 38,4 & 36,3 \\
\hline Ed.Superior & 4,6 & 32,1 & 27,8 \\
\hline
\end{tabular}

Finalmente, se sabe que cada vez que la madre supera un nivel educacional, el estudiante (su hijo) obtendrá en promedio 5.182181 más en su rendimiento de lenguaje. El problema es que - nuevamente - para los sectores rurales, la mayoría de las madres solamente han cursado educación básica (76\% frente al 36\% nacional) y en el ámbito opuesto, una proporción ínfima ha cursado estudios superiores $(4,6 \%$ anteel $28 \%$ nacional), dando cuenta de la estructura histórica de inequidades que la sociedad chilena ha sustentado por décadas, cuyos impactos resultan difíciles de mitigar por la acción directa del establecimiento escolar, si no se refuerzan sus estrategias educativas y, paralelamente, se interviene la comunidad con programas efectivos que nivelen oportunidades a la población de estudiantes del medio rural.

Las variables de primer nivel aportan interesantes posibilidades, enmarcadas en aquello que las teorías vigentes pueden explicar sobre los resultados educacionales, y evidenciando que muchas de estas variables pueden ser corregidas con políticas públicas que mostrarían alta y rápida eficiencia e impacto en el medio rural. El sentido se busca corregir aquellas "inequidades naturales" actuando sobre variables que pueden tener impactos positivos, como las que señaladas.

En primer lugar, hay clara evidencia de la importancia de la educación de la madre en los logros de sus hijos, por ende la necesidad de insistir en programas de nivelación de competencias educacionales para adultos - esencialmente las madres 
- redituará directamente en los logros de sus hijos, por lo que debiesen ser implementados con mayor urgencia en el medio rural, sin perjuicio debiesen plantearse de igual manera en aquellas localidades que lo ameriten.

Mediante esta acción debiesen tender a reducirse esas inequidades atávicas entre la población que han marcado generacionalmente las diferencias de resultados escolares entre los estudiantes del medio rural y del urbano. Esto debiese complementarse con tres medidas simples y eficaces para el medio rural: (i) Aumento de la cobertura de la educación preescolar, (ii) políticas de reducción de la repitencia asociadas al aumento de los aprendizajes, y (iii) mayor acceso a bienes culturales. Medidas que pueden ser eficientes - ciertamente - después del apoyo brindado a las madres, pues todas ellas aportarán a nivelar las condiciones de desempeño.

Adicionalmente dos aspectos importantes para el medio rural: primero potenciar las relaciones entre docentes y estudiantes como estímulo para los aprendizajes y, segundo, analizar la situación de los fuertes diferenciales de rendimientos por género y precisar la participación de variables culturales y socioeconómicas en este proceso.

\section{Variables de segundo nivel}

Las variables de segundo nivel se relacionan con las características de los establecimientos educacionales. Los principales resultados alcanzados en tres aspectos claves son:

\section{Tabla XI}

Dependencia del establecimiento

\begin{tabular}{|c|c|c|c|}
\hline \multirow[b]{3}{*}{ Público } & \multicolumn{2}{|c|}{ Zona/ área } & \multirow[b]{2}{*}{ Total } \\
\hline & Rural & No Rural & \\
\hline & 63,6 & 34,9 & 43,1 \\
\hline No Público & 36,4 & 65,1 & 56,9 \\
\hline
\end{tabular}

Los estudiantes de establecimientos de dependencia no pública obtendrán en promedio 13.361241 puntos más en sus pruebas de lenguaje. Sin embargo, al revés de la tendencia nacional, que da cuenta de un $57 \%$ de este tipo de colegios, en el sector rural, sólo un $36 \%$ de estos establecimientos es de dependencia nopública. 
Tabla XII

Presión de los padres hacia la escuela

\begin{tabular}{|lccc|}
\hline & \multicolumn{2}{c|}{ Zona/ área } & \\
\cline { 2 - 3 } & Rural & No rural & Total \\
\cline { 2 - 3 } Sin presión & 100,0 & 75,0 & 77,9 \\
\cline { 2 - 3 } Con presión & 0,0 & 25,0 & 22,1 \\
\hline
\end{tabular}

De igual forma, cuando se analiza el nivel de presión del apoderado a las escuelas, entendida como la atención expresa de los padres respecto del hacer del establecimiento, pese a que la tendencia global del país muestra una baja presión (22\%), en las zonas rurales claramente no existe o no se registra. La presión de los padres puede tener distintos impactos, pero desde el punto de vista de la rendición de cuentas es positiva, en tanto requiere que los padres tengan los conocimientos indispensables para ejercer una función fiscalizadora apropiada ala materia en debate.

\section{Tabla XIII}

El establecimiento selecciona estudiantes

\begin{tabular}{|c|c|c|c|}
\hline & \multicolumn{2}{|c|}{ Zona/ área } & \multirow{3}{*}{$\begin{array}{l}\text { Total } \\
69,2\end{array}$} \\
\hline & Rural & No rural & \\
\hline Sin selección & 100,0 & 58,6 & \\
\hline Con selección & 0,0 & 41,4 & 30,8 \\
\hline
\end{tabular}

Finalmente, la revisión del componente selección de estudiantes por rendimiento académico que realizan los establecimientos muestra que en general un 31\% lo lleva a cabo. Sin embargo, para las zonas rurales no existe ningún tipo de selección por rendimiento, este efecto segregador se traslada a los resultados en la prueba de Lenguaje Pisa 2009. Un estudio sobre movilidad diaria de estudiantes en Chile (DONOSO; ARIAS, 2011, 2013) da cuenta que en algunos territorios urbano marginales los estudiantes de menor rendimiento - que son a su vez los de menor capital social - se trasladan hacia los liceos rurales, confirmando con ello estos resultados, producto de la no selección ante los otros establecimientos que efectivamente seleccionan por este criterio y prescinde de los estudiantes con menor rendimiento. En síntesis, los aspectos reseñados confirman que hay algunas inequidades relevantes en este nivel, esencialmente referidas a establecimiento, localización y actitud de los padres, que se transforman - finalmente - en diferencias sobre los resultados educacionales reportados en el Pisa 2009. 


\section{Consideraciones finales}

La revisión llevada a cabo da cuenta que en casi todas aquellas variables que resultaron ser estadísticamente significativas, al momento de predecir los puntajes Lenguaje Pisa 2009, el sector rural presenta características que desfavorecen la obtención, en promedio, de puntajes más altos respecto a otros sectores urbanos como las ciudades o las metrópolis.

El análisis de los factores netamente académicos prioriza la importancia de la educación preescolar en el rendimiento futuro de los estudiantes, afectando más al área rural por presentar una menor cobertura y asistencia de estudiantes a este nivel de enseñanza. Un segundo factor indicado es el peso de la madre en los resultados educacionales de sus hijos, si bien ello es consistente con lo sabido, insiste en la necesidad de invertir en educación de las madres actuales, así como de las estudiantes mujeres para reducir el impacto negativo de esta variable en el medio rural, eso implica un diseño coherente de políticas educativas de corto y mediano plazo para potenciar este factor que es determinante.

En forma concomitante, las mejorías en este ámbito incidirían en la repitiencia de curso que es un factor aún no resuelto adecuadamente en el medio rural y que requiere de políticas de afirmación positiva en esta materia, que reduzcan el mayor ámbito de inequidad del sistema educacional que es este (después del analfabetismo). Estas políticas deben implicar: mejorías en retención de estudiantes que impacten la tasa de éxito oportuno, y esencialmente en logros de aprendizaje pues, en caso contrario, las inequidades serán reducidas solo formalmente.

Entendiendo que se trabaja con un problema complejo, las inequidades históricas se tornan invisibles, pues están siempre allí y han sido naturalizadas, en lugar de comprenderlas como el producto de políticas que han acentuado la inequidad o no la han corregido. En este plano es indispensable realizar mayores esfuerzos para corregir las inequidades que se han presentado históricamente, y que han dejado a los estudiantes rurales en una situación de postergación, mediante el diseño e implementación de medidas compensatorias más eficientes, cuyas pistas se identifican - al menos para un debate de mayor profundidad - a partir de lo expuesto alo largo de este trabajo.

A su vez, los establecimientos educacionales son claves, porque en el sector rural existe una clara preeminencia del sector público, que atiende a la población más vulnerable y, por ende, la que presenta mayores dificultades para alcanzar buenos resultados, confirmándose esta hipótesis, avalada además con las menores oportunidades que ofrece la sociedad a quienes viven en el medio rural.

Dos aspectos son de importancia para comprender este fenómeno. Uno, que los procesos de selección de estudiantes, abiertos y encubiertos, terminan asignando 
los menos aventajados y de mayor costo social al sector público, y segundo no se han implementado políticas eficientes de compensación de inequidades que apunten en forma directa resolver estas diferencias. A modo de ejemplo: la retención de estudiantes y promoción oportuna no tienen la atención debida en el país, pese a su gran impacto, como tampoco lo tienen los problemas de analfabetismo y baja escolaridad de la población adulta rural, que registran un impacto positivo en los aprendizajes de los estudiantes, en especial cuando se trata de sus madres.

La revisión de las variables que muestran impactos en esta materia da cuenta que con políticas públicas debidamente direccionadas podrían cerrarse en un plazo prudente las brechas de logro que se registran en la actualidad, haciendo más equitativa la sociedad. De paso, aprovechar algunas ventajas como es la posibilidad de sustentar mejores resultados escolares a partir de la relación docente/estudiante, lo que implica debiesen revisarse estas variables y proponerse estrategias en diversos planos que apoyen la consecución de los logros esperados.

\section{Notas}

1. Ejemplos de esta clase de programas son el P-900 - iniciativa gubernamental que apoyó a las 900 escuelas de enseñanza primaria con más bajos resultados de aprendizaje del país (según la medición de las pruebas nacionales en lenguaje y matemáticas denominadas como prueba Simce) - y el programa de Mejoramiento de la Calidad y Equidad de la Educación (Mece) para la educación Básica (primaria) urbana y rural.

2. Hay ejemplos de propuestas parciales, la más importante por su magnitud puede ser el Programa Mece Básica Rural, que consistió en una iniciativa con recursos del Banco Mundial implementada en la primera mitad de la década de los 90', intervención que dio cuenta de varias estrategias en las escuelas más pequeñas (uni, bi y tridocente), que fue descontinuada, quedando vigente solo los microcentros docentes.

3. Dado que se trata del primer acercamiento al tema en cuestión, bien vale la pena pasar por alto esta limitación, que además no resulta subsanable a partir de la información existente.

\section{Referencias}

AHUMADA, J. En vez de la miseria. Santiago de Chile: Editorial del Pacífico, 1958.

ALSPAUGH, J.W.; HARTING, R.D. Transition effects of school grade-level organization on student achievement. Journal of Research and Development in Education, v. 28, n. 3, p. 145-149, 1995.

BEYER, H.; VELASCO, C. Una educación pública más efectiva: ¿los árboles no dejan ver el bosque?. En: BELLEI, C.; CONTRERAS, D .; VALENZUELA, J.P. (Ed.). Ecos de la revolución pingüina. Avances, debates y silencios de la reforma educacional. Santiago de Chile: Universidad de Chile-Unicef, 2010. p. 183-224. 
BOURDIEU, P.; PASSERON J.C. La Reproduction. Elements pour une théorie du systeme d'enseignement. Paris: Du Minuit, 1970.

CASTRO, B.; GAVIRIA, M. Clima escolar y comportamientos psicosociales en niños. Revista de la Facultad Nacional de Salud Pública, Colombia, v. 23, n. 2, p. 59-69, jul.-dic. 2005.

CARTWRIGHT, F.; ALLEN, M. Understanding the urban-rural reading gap. Ottawa: Statistics Canada, 2002.

CAVIERES, E. The class and cultural - based exclusion of the Chilean neoliberal educational reform. Educational Studies, n. 47, p. 111-132, 2011.

CERVINI, R Variación de la equidad en resultados cognitivos y no cognitivos de la educación media en Argentina. Redie - Revista Electrónica de Investigación Educativa, v. 7, n. 1, 2005. Disponible en: <www.redie.com>. Consultada en: dic. 2011.

COLEMAN, J.S. et al. Equality of educational opportunity, Washington, DC: Government Printing Office, 1966. (2 v.).

DONOSO, S. Financiamiento y gestión de la educación pública chilena en los años 90. Cadernos de Pesquisa, São Paulo, v. 36, n. 127, p. 151 -172, jan./mar. 2006.

DONOSO, S.; ARIAS, O. Diferencias de escala en los sistema de educación pública en Chile. Ensaio, Rio de Janeiro, v. 19, n. 71, p. 283 -307, abr./jun. 2011.

DONOSO, S.; ARIAS, O. Desplazamiento cotidiano de estudiantes entre comunas de Chile: Evidencia y recomendaciones de política para la nueva institucionalidad de la educación pública en Chile. Eure, v. 39, n. 116, p. 35 -54, 2013.

DRAGO, J.L; PAREDES, R. La brecha de calidad en la educación chilena. Revista Cepal, Santiago de Chile, n. 104, ago. 2011.

ESPINDOLA E.; LEÓN, A. La deserción escolar en América Latina: un tema prioritario para la agenda regional. Revista Iberoamericana de Educación, n. 30, p. 39-62, 2002.

GOLDSTEIN, H.; SAMMO, P. The influence of secondary and junior schools on sixteen year examination performance: a cross-classified multilevel analysis. School Effectiveness and School Improvement, v. 8, n. 2, p. 219-230, 1997.

HAMUY, E. El problema educacional del pueblo de Chile. Santiago de Chile: Editorial del Pacífico, 1961.

HSEIH, C.-T.; URQUIOLA, M. When school competes, how they compete? An assessment of Chile's nation wide school voucher program. Washington, DC: World Bank's Development Group, 2002. 
Inequidades invisibles en la educación Chilena...

HSIEH, C.-T.; URQUIOLA, M. The effects of generalized school choice on achievement and stratification: evidence from Chile's voucher program. Journal of Public Economics, Amsterdam, n. 90, 2006.

INSTITUTO NACIONAL DE ESTADÍSTICAS (INE). Compendio Estadístico 2010. Instituto Nacional de Estadísticas, 2010. Disponible en: <http://www.ine.cl/canales/menu/ publicaciones/compendio_estadistico/compendio_estadistico2010.php> Accedido el: 5 mar. 2012.

INSTITUTO NACIONAL DE ESTADÍSTICAS (INE). Compendio Estadístico 2012. Instituto Nacional de Estadísticas. Disponible en: <http://www.ine.cl/canales/menu/ publicaciones/compendio_estadistico/compendio_estadistico2012.php> Accedido el: 6 mar. 2012.

ILLANES, M.A. Ausente señorita. El niño chileno, la escuela para pobres y el niño chileno. Chile 1890 -1990. Santiago de Chile: Junta Nacional de Auxilio Escolar y Becas, 1991.

LABARCA, A. Historia de la enseñanza en Chile. Santiago de Chile: Imprenta Universitaria, 1939.

LOPEZ, N.; PEREYRA, A.; SOURROUILLE, F. Disparidades urbanas y rurales en América Latina. Algunas de sus implicancias en el acceso a la educación. Buenos Aires: IIEPUnesco, 2007. Disponible en: <http://unesdoc.unesco.org/images/0015/001555/155540s. pdf> Accedido el: 12 jun. 2012.

MIZALA, A.; TORCHE, F. Bringing the schools back in: the stratification of educational achievement in the Chilean voucher system. Int. Jornal of Educational Development, n. 32 p. 132-144, 2012.

OECD. Estudio Económico de Chile. París: Organización para la Cooperación y el Desarrollo Económico, 2010.

OYARZÚN, M.E.G.; MIRANDA, E.B. La economía rural en Chile: entre la pobreza y el desarrollo. Revista Estudios de Economía Aplicada, v. 29, n. 1, p. 31-56, 2011.

PAREDES, R.; PAREDES, V. Chile: rendimiento académico y gestión de la educación en un contexto de rigidez laboral. Revista Cepal, n. 99, p. 119-131, 2009.

SALAS, D. El problema nacional. Santiago de Chile: Facultad de Filosofía y Educación, Universidad de Chile, 1917.

SERRANO, S.; PONCE DE LEÓN, M.; RENGIFO, F. Historia de la Educación en Chile (1810- 2010) - Tomo 1: Aprender a leer a y escribir (1810 -1880). Santiago de Chile: Editorial Taurus, 2012a. 
SERRANO, S.; PONCE DE LEÓN, M.; RENGIFO, F. Historia de la Educación en Chile (1810- 2010) Tomo 1I. La Educación nacional, 1880 -1930. Santiago de Chile: Editorial Taurus, 2012b.

TEDESCO, J.C. Educación y justicia social en América Latina. Buenos Aires: Fondo de Cultura Económica, Universidad de San Martín, 2012.

TREVIÑO, E.; DONOSO, F. Agrupación de escuelas para intervenciones de política: análisis del caso chileno. Santiago de Chile: Universidad Diego Portales, Facultad de Educación, 2010.

Recebido em 3 de setembro de 2012.

Aprovado em 30 de julho de 2013. 
